\title{
Eficacia de la azatioprina en la enfermedad ocular inflamatoria no infecciosa resistente a tratamiento esteroidal sistémico
}

\author{
Miguel Cuchacovich ${ }^{1,2}$, Patricio Pacheco ${ }^{3}$, Gonzalo Díaz ${ }^{a}$, \\ Basilio Rojas ${ }^{5}$, Juan Stoppel ${ }^{5}$, G uillermo Merino ${ }^{4}$, \\ Juan Ignacio Verdaguer 5 , Juan Verdaguer ${ }^{5}$, \\ Francisco Villarroel ${ }^{5}$. \\ Role of Azathioprine in steroid \\ resistant non infectious ocular \\ inflammatory diseases
}

Background: Topical and systemic steroids are the first line of treatment of non infectious inflammatory ocular disease. Immunosuppresants are reserved as a second line treatment. Aim: To evaluate the role of Azathioprine (AZA) as a coadyuvant immunosuppressive treatment for non infectious ocular inflammatory diseases (OIDs) resistant to systemic steroid therapy in a retrospective, noncomparative interventional case series. Patients and methods: Patients using oral Prednisone due to an active or recurrent OID, without clinical response, and not receiving any other immunosuppressive treatment were studied. A standard protocol of oral Prednisone $(0.5 \mathrm{mg} / \mathrm{kg} /$ day) and oral AZA (2-3 mg/kg/day) during one year was used. Ocular and systemic monthly evaluations were done including relapse rate, steroid dosage, inflammatory score and visual acuity. Results: Thirty patients (10 male) aged 18-75 years (mean 44 years) were studied. Three had bilateral anterior uveitis, one had pars planitis, four had diffuse uveitis, eight Vogt-Koyanahi-Harada syndrome, three Behçet's disease, three necrotizing scleritis and eight had retinochoroidopathy. A complete initial response was observed in 26 patients (87\%). The time of response was between 1 to 6 months (mean 2.65 months). Seventeen percent of these had a relapse 6 to 12 months after AZA was started. In $61 \%$, visual acuity improved. The ocular inflammatory score decreased in $86.5 \%$. Eleven patients had mild controlled side effects that did not require discontinuation of AZA. Conclusions: Combined systemic steroid and oral AZA therapy is safe and effective in controlling steroid resistant non infectious inflammatory ocular diseases (Rev Méd Chile 2007; 135: 702-7).

(Key words: Azathioprine; Prednisone; Uveitis; Vogt-Koyanagi-Harada Syndrome)

\footnotetext{
Recibido el 16 de agosto, 2006. Aceptado el 7 de noviembre, 2006.

${ }^{1}$ Sección de Reumatología, Departamento de Medicina, Hospital Clínico de la Universidad de Chile, Santiago, Chile. ${ }^{2}$ Sección de Reumatología, Departamento de Medicina, Clínica Las Condes. ${ }^{3}$ Departamento de Oftalmología, Hospital Clínico de la Universidad de Chile. ${ }^{4}$ Centro Oftalmológico Luis Pasteur. ${ }^{5}$ Fundación Oftalmológica Los Andes.

anterno de Medicina, Universidad de Chile.
}

Correspondencia a: Dr. Miguel Cuchacovich. San Pío X No 2460,

Oficina 607. Providencia, Santiago, Chile. Fono: 2048821. Fax:

2312395. E mail: mcuchacovich@redclinicauchile.cl 
$\mathrm{E}^{1}$ tratamiento esteroidal tópico y sistémico constituye la primera alternativa en el manejo de la enfermedad ocular inflamatoria no infecciosa (EOI). Los inmunosupresores se utilizan como segunda línea, frente a la ineficacia terapéutica de los esteroides o cuando aparecen efectos colaterales $^{1,2}$.

Los agentes citotóxicos más utilizados en el tratamiento de la EOI son ciclofosfamida, ciclosporina A y metotrexato ${ }^{3,4}$.

Muchos pacientes con EOI se encuentran entre la tercera y cuarta década de la vida. El tratamiento con ciclofosfamida conlleva el riesgo potencial de amenorrea e infertilidad en esta población ${ }^{5}$. La ciclosporina A es un fármaco efectivo en el control de la inflamación ocular en pacientes con $\mathrm{EOI}^{6,7}$, sin embargo, su costo es elevado y la mayoría de los pacientes no pueden acceder a este fármaco. A la fecha, existen escasas publicaciones en la literatura mundial sobre la eficacia de la azatioprina (AZA) en pacientes con uveítis $^{8}$. AZA es un fármaco menos tóxico que la ciclofosfamida y más económico que la ciclosporina A, por lo que podría ser considerado de primera elección en el manejo de la EOI resistente a esteroides.

Por lo anterior y con el fin de evaluar la eficacia terapéutica de la AZA, hemos estudiado retrospectivamente un grupo de pacientes chilenos portadores de EOI resistente a tratamiento esteroidal, los que fueron tratados con una combinación de esteroides sistémicos y AZA.

\section{MATERIAL Y MÉTODO}

Pacientes. Se revisaron las fichas clínicas de los pacientes portadores de una EOI resistente a esteroides que consultaron en los departamentos de oftalmología de los centros participantes (Hospital Clínico de la Universidad de Chile, Fundación Oftalmológica Los Andes y Clínica Las Condes) o en la consulta privada de uno de los autores (MC), que recibieron tratamiento asociado con AZA. Se obtuvo consentimiento informado de todos los pacientes antes de iniciar el tratamiento inmunosupresor.

Los criterios de inclusión para el registro de estos pacientes fueron: pacientes que estuvieran recibiendo al menos $30 \mathrm{mg} /$ día de prednisona y que continuaban con EOI activa o pacientes que hubieran respondido, pero que requirieran de una dosis $\geq 20 \mathrm{mg} /$ día para permanecer inactivos o que presentaran efectos colaterales severos con el tratamiento esteroidal. Ningún paciente podía haber recibido tratamiento inmunosupresor durante los 12 meses previos al tratamiento con AZA.

El diagnóstico específico de cada EOI se basó en los criterios de clasificación del grupo internacional de estudios de uveítis ${ }^{9}$.

En todos los pacientes se realizó un detallado examen clínico y de laboratorio con el fin de descartar la presencia de infecciones activas (VDRL-FTA ABS, IgM e IgG para toxoplasmosis y PPD) y un estudio inmunológico (factor reumatoide, anticuerpos antinucleares, HLA B 27, ANCA, anticuerpos anti ENA, anticuerpos anticardiolipinas y niveles de C3 y C4). Se realizó también un hemograma y VHS, perfil bioquímico y examen de orina completo al ingreso al estudio y luego mensualmente durante el seguimiento.

Los pacientes que recibieron tratamiento con AZA tenían función hepática y renal normal y ausencia de patologías hematológicas, inmunodeficiencias o de enfermedad neoplásica.

Protocolo de tratamiento. Los pacientes con EOI activa fueron tratados con una combinación de prednisona y AZA por uno de los autores (MC), utilizando dosis convencionales de acuerdo a un esquema internacionalmente aceptado ${ }^{8}$.

La dosis inicial de prednisona fue de $0,5 \mathrm{mg} / \mathrm{kg} /$ día durante 4 semanas. En caso que el paciente experimentara mejoría clínica, se continuaba con una reducción progresiva durante los 3 meses siguientes, hasta alcanzar una dosis de mantención que oscilaba entre 5 y $10 \mathrm{mg} /$ día. Si la EOI continuaba activa más allá de las 4 semanas iniciales, entonces la dosis de prednisona era reducida en forma más lenta. La prednisona se mantuvo por un año en una dosis de 5 a $10 \mathrm{mg} /$ día.

La AZA fue administrada en dosis de $2-3 \mathrm{mg} / \mathrm{kg} /$ día por un año. Los pacientes en tratamiento inmunosupresor fueron evaluados mensualmente con un examen clínico detallado y con hemograma y VHS, creatininemia, transaminasas y examen químico y citológico de orina.

La AZA fue suspendida si el recuento de leucocitos era inferior a 3.000 por $\mathrm{mm}^{3}$, si el recuento de plaquetas era menor $100.000 / \mathrm{mm}^{3}$ o si la hemoglobina era menor de $7 \mathrm{~g} / \mathrm{dl}$. 
En el análisis de eficacia, cada paciente fue utilizado como su propio control. Se comparó entre el período pretratamiento, el período de tratamiento con AZA y el estado de la enfermedad al finalizar la terapia si el tratamiento debía ser discontinuado por algún motivo. Se registró la tasa de recaídas (número de eventos por año) antes del tratamiento con AZA y se comparó con la del período de tratamiento con AZA. Se definió una recaída como un aumento de la celularidad a la biomicroscopia o la aparición de lesiones frescas al fondo de ojo, las que generalmente se acompañaban de disminución de la agudeza visual.

Los pacientes que presentaban severa opacidad del cristalino, que impedía una adecuada evaluación, fueron excluidos del estudio.

Se registró la dosis de esteroides promedio que cada paciente recibía antes de iniciar la AZA y se la comparó con aquella obtenida durante el tratamiento con AZA. La eficacia clínica durante y al finalizar el tratamiento con AZA, fue evaluada mediante el cambio en el score inflamatorio y en la escala de Snellen de agudeza visual. Un cambio de al menos dos líneas en la escala de Snellen, fue considerado como significativo. El score inflamatorio fue calculado usando una escala de morbilidad visual, de acuerdo a los criterios del grupo internacional de estudios de uveítis ${ }^{9,10}$ y cada ojo fue evaluado en forma separada.
Se analizó también la aparición de efectos colaterales con este esquema de tratamiento.

Estadística. Se analizaron las diferencias entre la agudeza visual pre y postratamiento mediante un test $t$ de Student para 2 muestras ${ }^{11}$.

Las diferencias observadas entre la agudeza visual pre y postratamiento fueron evaluadas por el test $\mathrm{t}$ de Student para 2 muestras de iguales varianzas.

Se consideró estadísticamente significativo, un valor de $\mathrm{p}<0,05$.

\section{Resultados}

Características de los pacientes. Se obtuvieron datos completos de las fichas de treinta pacientes (10 hombres y 20 mujeres), con un rango de edad que oscilaba entre 18 y 75 años (mediana 44 años), portadores de EOI crónica. La Tabla 1 muestra las diferentes categorías diagnósticas de los pacientes. La Tabla 2 muestra las complicaciones secundarias a la EOI al inicio del tratamiento con AZA.

Curso clínico y respuesta al tratamiento. Se observó una remisión completa inicial en 51 ojos (26 pacientes, 87\%) y una respuesta parcial o ausencia

Tabla 1. Tipos de enfermedad ocular inflamatoria tratadas con azatioprina

\begin{tabular}{|lrrr|}
\hline Tipo & Masculino & Femenino & Total \\
\hline Uveitis & & & \\
$\quad$ Anterior & 0 & $3(6)$ & $3(6)$ \\
$\quad$ Pars Planitis & 0 & $1(2)$ & $1(2)$ \\
$\quad$ Difusa & $1(2)$ & $3(6)$ & $4(8)$ \\
Enfermedad VKH & $1(2)$ & $7(14)$ & $8(16)$ \\
Śndrome de Behçet & $3(6)$ & $0(0)$ & $3(6)$ \\
Escleritis & $1(2)$ & $2(3)$ & $3(5)$ \\
Coroidoretinopatía & & & $2(4)$ \\
$\quad$ Birdshot & $0(0)$ & $2(4)$ & $2(4)$ \\
$\quad$ Serpiginosa & $1(2)$ & $1(2)$ & $4(8)$ \\
$\quad$ Vasculitis retinal primaria & $3(6)$ & $1(2)$ & $30(59)$ \\
Total & $10(20)$ & $20(39)$ & \\
\hline
\end{tabular}

( )= número de ojos afectados. 
Tabla 2. Complicaciones oculares asociadas a la enfermedad ocular inflamatoria

\begin{tabular}{|lc|}
\hline Complicaciones & Número de ojos \\
\hline Edema macular cistoide & 8 \\
Catarata & 8 \\
Glaucoma secundario & 5 \\
Membrana epirretinal & 3 \\
Aneurisma retinal secundario & 1 \\
Neuritis óptica & 1 \\
\hline
\end{tabular}

Tabla 3. Respuesta clínica a la azatioprina al final del seguimiento

\begin{tabular}{|lrc|}
\hline Tipo de respuesta & $\mathrm{n}$ & $\%$ \\
\hline Respuesta completa & 21 & $(70)$ \\
Recaída & 7 & $(17)$ \\
Sin respuesta & 4 & $(13)$ \\
\hline
\end{tabular}

\section{Tabla 4. Evolución de la agudeza visual en pacientes portadores de enfermedad ocular inflamatoria tratados con azatioprina}

\begin{tabular}{|lcrc|}
\hline Agudeza visual & $\begin{array}{c}\text { Inicial } \\
\mathrm{n}\end{array}$ & $\begin{array}{c}\text { Final } \\
\mathrm{n}\end{array}$ & $\mathrm{P}$ \\
\hline$>0,8$ & 10 & 34 & $<0,001$ \\
$0,5-0,8$ & 18 & 8 & NS \\
$<0,5$ & 31 & 17 & NS \\
\hline
\end{tabular}

$\mathrm{N}=$ número de pacientes.

NS =no significativo.

de respuesta en 8 ojos (4 pacientes, 13\%). El inicio de la respuesta clínica al tratamiento con AZA osciló entre 1 y 6 meses (promedio de 2,65 meses). La mayonía de las complicaciones asociadas a la EOI se resolvieron en los pacientes respondedores a la AZA, constatándose una reducción de la actividad inflamatoria y una mejoría de la agudeza visual en la mayoría de ellos. Veintiuno (70\%) de los 26 pacientes que alcanzaron una remisión inicial, se mantienen en remisión clínica a la fecha, sin evidencias de recaída en su último control, con una media de seguimiento de 37,5 meses. Cinco pacientes (17\%) presentaron una recaída (Tabla 3 ).
Tasa de recaídas. Cinco pacientes (10 ojos, 17\%) presentaron una recaída después de haber alcanzado una remisión inicial. Esto ocurrió entre los 6 y 12 meses de iniciado el tratamiento con AZA (mediana de 7,2 meses). Sin embargo, la inflamación asociada a la recaída fue de menor intensidad que el grado de inflamación pretratamiento. En 3 casos se asoció ciclosporina A (5 mg/ $\mathrm{kg} /$ día). Un paciente respondió a la adición de ciclosporina $\mathrm{A}$, con reducción de la actividad inflamatoria y mejoría de la agudeza visual. Los otros 2 pacientes no respondieron a la ciclosporina A. En un paciente se aumentó la dosis de esteroides sistémicos, mientras que en otro se adicionó triamcinolona intra vítrea. En ambos casos se logró sólo un control transitorio de la inflamación ocular.

Dosis de esteroides. Antes de iniciar el tratamiento con AZA, la dosis promedio de prednisona era de 45,1 $\mathrm{mg} /$ día. Al mes de tratamiento, la dosis promedio de prednisona se había reducido a 25,9 mg/día, a los 3 meses a $14 \mathrm{mg} /$ día y a los 6 meses a 5,1 mg/día. Esta reducción se observó en 26 casos (86\%) a los 6 meses de tratamiento, sin embargo, a partir de ese momento se observó una recaída en 5 casos, como fue descrito anteriormente.

Inflamación ocular. La magnitud de la inflamación intraocular fue cuantificada para cada ojo antes de iniciar el tratamiento con AZA y luego mensualmente durante el tratamiento inmunosupresor. Cincuenta y un ojos disminuyeron la actividad inflamatoria (86,5\%), mientras que en 8 ojos (13,5\%) no se observó disminución de la inflamación.

Agudeza visual. La agudeza visual con corrección fue medida con una escala de Snellen antes de iniciar el tratamiento con AZA y luego mensualmente durante el tratamiento. Los 59 ojos comprometidos (30 pacientes) fueron usados como sus propios controles. Treinta y seis ojos (61\%) mejoraron su agudeza visual (en al menos 2 líneas de la escala de Snellen) al final del seguimiento, 12 ojos (20\%) mantuvieron su agudeza visual y 11 ojos (19\%) disminuyeron su agudeza visual (Tabla 4). Se observó una mejoría significativa de la agudeza visual al final del tratamiento con AZA ( $p<0,01$ ).

Efectos colaterales. La Tabla 5 muestra los efectos colaterales presentados durante el estudio. En 4 
Tabla 5. Efectos colaterales durante el tratamiento con azatioprina

\begin{tabular}{|lcc|}
\hline Efecto colateral & $\begin{array}{c}\text { Número de pacientes } \\
\text { afectados }\end{array}$ & $\begin{array}{c}\text { Número de pacientes } \\
\text { que suspendieron tratamiento }\end{array}$ \\
\hline Gastrointestinal* & 2 & 0 \\
Herpes zoster & 2 & 0 \\
Cefalea & 2 & 0 \\
Leucopenia & 2 & 0 \\
Aumento de transaminasas & 2 & 0 \\
Infección urinaria & 1 & 0 \\
\hline
\end{tabular}

*(náuseas y vómitos).

pacientes se suspendió definitivamente la terapia con AZA, pero en ninguno fue debido a efectos colaterales graves. La suspensión se debió en todos ellos a ineficacia del tratamiento.

\section{DisCUSIÓN}

La azatioprina es un análogo imidazólico de la 6mercaptopurina. Este agente inmunosupresor ha sido utilizado en una gran variedad de patologías autoinmunes, incluyendo enfermedades reumatológicas, dermatológicas y enfermedades inflamatorias intestinales ${ }^{12}$.

Algunos reportes han demostrado su efectividad en diferentes EOI no infecciosas refractarias a esteroides como el penfigoide ocular, vasculitis retinal primaria, compromiso ocular del síndrome de Behçet, uveítis crónica o recurrente ${ }^{13}$ y miastenia gravis ocular ${ }^{14}$.

Los pacientes tratados en nuestra serie se encuentran, en su mayoría, en la edad media de la vida (promedio de 44 años); son personas activas, sin enfermedades sistémicas y con un predominio del sexo femenino (2:1), como es frecuente en este tipo de patologías.

Todos los pacientes de esta cohorte habían recibido esteroides por un período prolongado (promedio 13,3 meses), sin lograr un control clínico de su enfermedad antes de iniciar tratamiento con AZA, lo que ilustra la severidad del cuadro clínico.

La mayoría de los pacientes tratados (81\%) mejoraron o estabilizaron su agudeza visual y presentaron una significativa disminución de la inflamación ocular (86,5\% de los ojos comprometidos). Estos resultados permitieron reducir la dosis de esteroides en $87 \%$ de los casos, a los 6 meses de tratamiento. Este hecho es muy significativo, ya que permitió, a su vez, reducir las severas complicaciones del tratamiento esteroidal sistémico prolongado.

Otro hecho importante es el bajo perfil de toxicidad de la AZA. Todos los efectos colaterales fueron tratados exitosamente y ningún paciente debió discontinuar la AZA en forma definitiva debido a esta causa.

Nuestros resultados son concordantes con otro estudio que demuestra que la AZA es un fármaco eficaz en el control de la EOI, con un perfil de toxicidad menor que el de la ciclofosfamida ${ }^{13}$.

La necesidad de desarrollar nuevos inmunosupresores más efectivos y menos tóxicos en las enfermedades autoinmunes, es un problema importante y no resuelto a la fecha. Sin embargo, nuestros resultados demuestran que la AZA parece ser un agente eficaz y con un perfil de toxicidad aceptable en un porcentaje importante de pacientes con EOI. La escasez de publicaciones sobre la eficacia de la AZA en la EOI, podría indicar que este fármaco pudiera estar siendo subutilizado en la práctica clínica.

Nuevos estudios prospectivos sobre la eficacia de la AZA en cada categoría diagnóstica de EOI por separado, deberán ser realizados a futuro, con el fin de detectar aquellas patologías que presentan una mejor respuesta clínica a este tratamiento. 


\section{REFERENCIAS}

1. Tamesis RR, Rodríguez A, Christen WG, Akova YA, MESSMER E, Foster CS. Systemic drug toxicity trends in immunosuppressive therapy of immune and inflammatory ocular disease. Ophthalmology 1996; 103: 768-75.

2. Santamaría J II. Steroidal agents: their systemic and ocular complications. Ocular Inflamm Ther 1983; 1: 19-26.

3. Nussenblatt RB, Palestine AG, Chan CC. Cyclosporin A therapy in the treatment of intraocular inflammatory disease resistant to systemic corticosteroids and cytotoxic agents. Am J Ophthalmol 1983; 96: 275-82.

4. Hemady R, Tauber J, Foster CS. Immunosuppressive drugs in immune and inflammatory ocular disease. Surv Ophthalmol 1991; 35: 369-85.

5. Wang CL, Wang F, Bosco JJ. Ovarian failure in oral cyclophosphamide treatment for systemic lupus erythematosus. Lupus 1995; 4: 11-4.

6. Vitale AT, Rodriguez A, Foster CS. Low-dose cyclosporin A therapy in treating chronic noninfectious uveitis. Ophthalmology 1996; 103: 365-74.

7. Cuchacovich M, Gatica H, Verdaguer Ji, Carreño MA, Viliarroel F, Verdaguer J. Tratamiento con dosis bajas de ciclosporina A en pacientes con enfermedad ocular inflamatoria de etiología no infecciosa. Rev Méd Chile 1999; 127: 277-85.
8. Greenwood AJ, Stanford MR, Graham EM. The role of azathioprine in the management of retinal vasculitis. Eye 1998; 12: 783-8.

9. Bloch-Michel E, Nussenblatt RB. International Uveitis Study Group recommendations for the evaluation of intraocular inflammatory disease. Am J Ophthalmol 1987; 103: 234-5.

10. Nussenblatt RB, Palestine AG, Chan CC, Roberge F. Standardization of vitreal inflammatory activity in intermediate and posterior uveitis. Ophthalmoly 1985; 92: 467-71.

11. Armitage P, BerRy G. The Statistic Inference, en: Armitage P, Berry G, eds. Statistics for the biomedical investigation. Madrid, Spain: Harcourt Brace, 1997, 111-32.

12. Corley CC JR, Lessner HE, Larsen WE. Azathioprine therapy of «autoimmune» diseases. Am J Med 1966; 41: 404-12.

13. Flores M, Gudino Pérez R, Ríos Prado R, Almedia Arvizu VM, Castrejón Vázquez MI, Ocampo aA. Comparative study of the treatment of autoimmune uveitis with prednisone and with cyclophosphamide and azathioprine. Rev Alerg Mex 2001; 48: 75-9.

14. Mee J, Paine M, Byrne E, King J, Reardon K, O’Day $\mathrm{J}$. Immunotherapy of ocular myasthenia gravis reduces conversion to generalized myasthenia gravis. J Neuroophthalmol 2003; 23: 251-5. 